\title{
ESPECIE NUEVA DE MILPIÉS DEL GÉNERO PODISCODESMUS (DIPLOPODA: POLYDESMIDA: CHELODESMIDAE) PARA LA HISPANIOLA
}

\author{
Antonio R. Pérez-Asso \\ Investigador Asociado, Museo Nacional de Historia Natural, Santo Domingo (MNHNSD), Plaza de la Cultura, \\ Santo Domingo, República Dominicana. \\ Hparaiso@caribe.net
}

\section{RESUMEN}

Se describe una especie nueva de chelodésmido del género Podiscodesmus para La Hispaniola. Este género se caracteriza por presentar gonopodos extremadamente pequeños y estructuras muy simples.

Palabras clave: Diplopoda, Polydesmida, Chelodesmidae, Podiscodesmus, especie nueva.

\section{ABSTRACT}

A new chelodesmid species belonging to the genus Podiscodesmus is described from Hispaniola. Extremely small and simple gonopods characterize this genus.

Key words: Diplopoda, Polydesmida, Chelodesmidae, Podiscodesmus, new species.

\section{INTRODUCCIÓN}

El género Podiscodesmus fue descrito por Loomis en 1941, en base a dos especímenes machos colectados en los alrededores del poblado de Sánchez, en la península de Samaná. La especie fue nombrada Podiscodesmus carinatus y era la única conocida de este género, considerado hasta ahora monotípico. En el mes de diciembre del año 2007 colecté numerosos ejemplares pertenecientes a esta especie, en áreas cercanas a la localidad tipo, lo que aumenta ampliamente su representación en colecciones.

Comparando a P. carinatus con especímenes colectados en la Sierra de Neiba en noviembre de 2006, observé semejanzas en cuanto al tamaño y forma de los gonopodos, por lo que consideré conveniente acomodar a estos ejemplares en el mismo género Podiscodesmus; sin embargo, vale resaltar que $P$. carinatus y la nueva especie que describo a continuación muestran estructuras corporales muy diferentes (Figs.1-2).

\section{MATERIALES Y MÉTODOS}

Se determinó la longitud total midiendo los ejemplares desde la cabeza hasta el ápice del epiprocto; el ancho se tomó al nivel del séptimo segmento. Todos los especímenes medidos fueron conservados cuidadosamente, quedando perfectamente aplanados, lo que facilita notablemente su estudio. De estas mediciones se presentan los valores medios y entre paréntesis los valores extremos.

Las ilustraciones se realizaron con cámara lúcida en un microscopio Wild MSD. Los especímenes estudiados se encuentran depositados en la colección ARPA (colección privada del autor), algunos paratipos se donaron a la colección del Museo Nacional de Historia Natural de Santo Domingo, República Dominicana.

\section{RESULTADOS}

Género Podiscodesmus Loomis

Podiscodesmus Loomis, 1941, Bull. Mus. Comp. Zool., 88: 56. Pérez-Asso y Pérez-Gelabert, 2001, Bol. S. E. A. 28: 75.

Diagnosis. Género de chelodésmido con gonopodos muy simples y extremadamente pequeños, los cuales no alcanzan el esternito del sexto segmento (Figs.5, 8). 


\section{Podiscodesmus neiba sp. nov.}

(Figs.1, 3-5).

Diagnosis. Dorso cubierto de macrosetas. Ángulo anterior de los paraterguitos con diente. Ángulo posterior de los paraterguitos de los segmentos no poríferos muy águdo. Los segmentos poríferos con callo prominente. Gonopodos según fig. 3 y 4. P. carinatus no posee macrosetas en el dorso, no presenta diente en el ángulo anterior de los paraterguitos, el ángulo posterior de los segmentos no poríferos es redondeado, y el callo porífero no es pronunciado.

Diagnosis. Dorsal surface with macrosetae. Anterior corner of each paraterguite with a tooth. Posterior corner of paraterguites at non poriferous segments, very acute. Poriferous segments with prominent pore callus. Gonopods according to Figs. 3 and 4. P. carinatus do not have macrosetae at dorsal surface, neither a tooth at the anterior angle of paraterguites; anterior angles at the non poriferours segments rounded; pore callus not noticeable.

Holotipo. Macho 6669; largo 26,7 mm., ancho 3,3 mm. Cabeza castaño oscuro. Collum y segmentos castaño oscuro con paraterguitos castaños. Pleuritos castaños; esternitos castaño claro. Antenas castaño rojizo; patas castañas. Epiprocto con la mitad anterior castaño oscuro y la mitad posterior castaño claro; hypoprocto y valvas anales castañas. Collum y segmentos del cuerpo con macrosetas en el dorso, ordenadas en tres hileras transversales más o menos alineadas (Fig.1). A continuación se presenta, entre paréntesis, el número de macrosetas de cada hilera en cada segmento del cuerpo: collum (6-6-7), segmento 2 (6-6-8), 3 (6-6-8), 4 (4-6-8), 5 (6-6-8), 6 (6-5-6), 7 (5-6-6), 8 (5-6-5), 9 (6-6-5), 10 (6-6-7), 11 (6-4-6), 12 (8-6-7), 13 (7-4-6), 14 (6-6-8), 15 (8-8-8), 16 (8-6-8), 17 (7-7-8), 18 (8-7-8), 19 (7-8-11). Segmentos poríferos $(5,7,9-10,12-13,15-19)$ sin macrosetas en el margen lateral de los paraterguitos. Segmentos no poríferos $(6,8,11,14)$ con dos macrosetas en el margen lateral de cada paraterguito. Callo porífero muy grande y alargado. Gonopodos muy pequeños (Fig.5), con el fémur simple, ensanchado en la base y estrechándose hacia el ápice; proceso prefemoral también simple, ensanchado hacia la región central y estrechándose hacia el ápice (Figs.3 y 4 ).
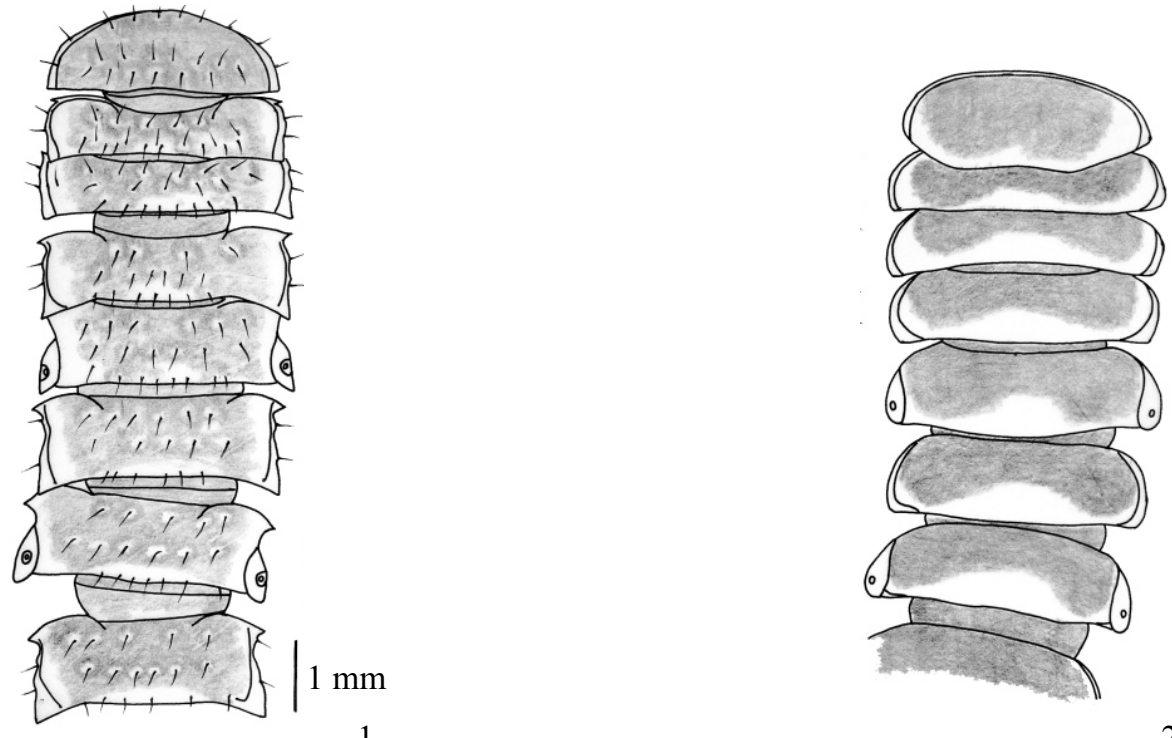


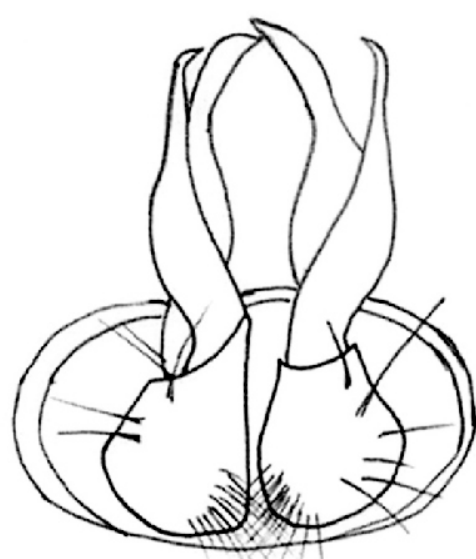

3
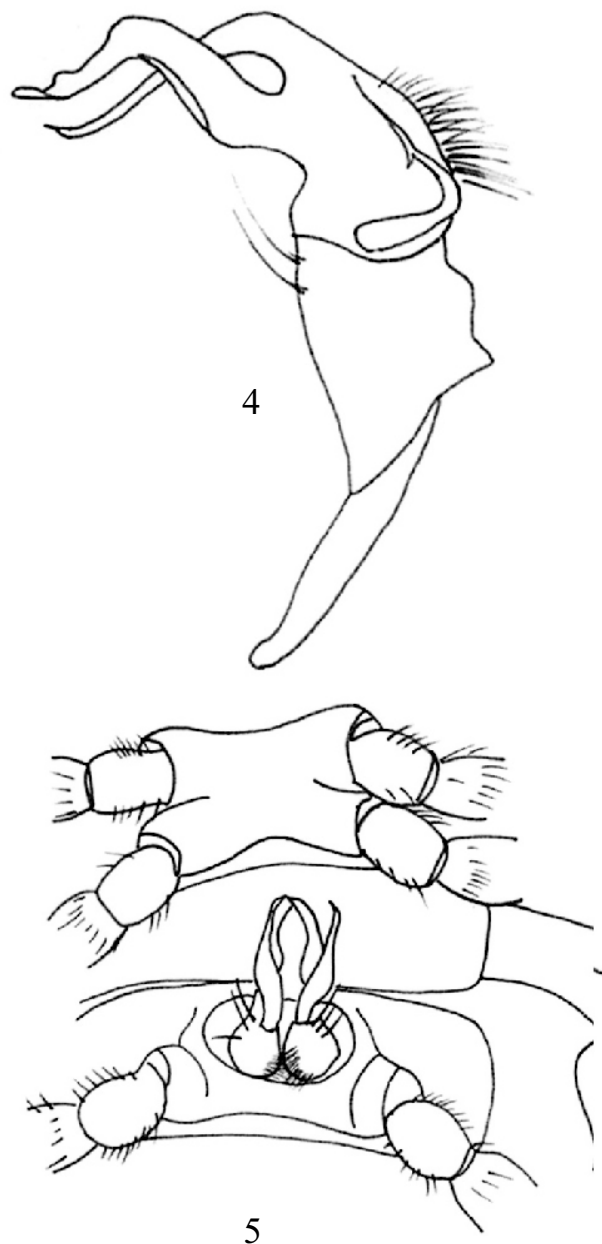

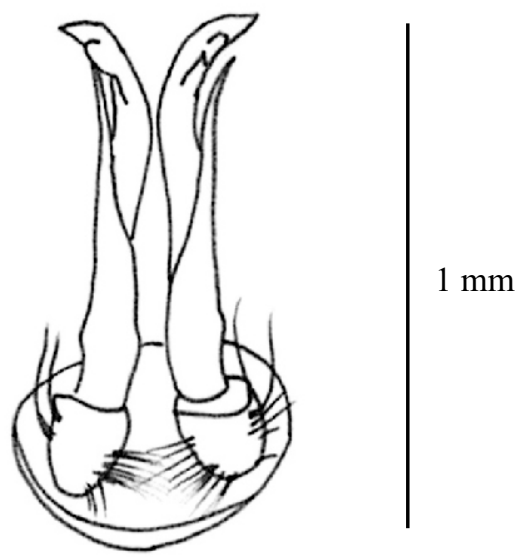

6
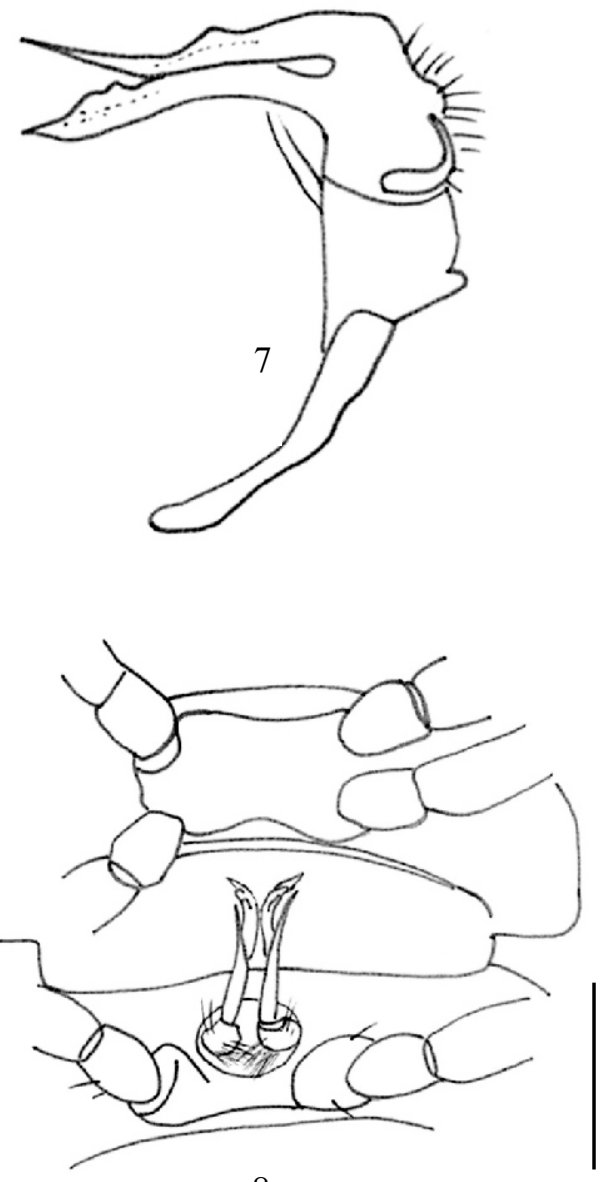

8 
Variabilidad. $\mathrm{N}$ representa el número total de ejemplares medidos. X representa el valor promedio. Los intervalos entre paréntesis muestran los valores mínimo y máximo. Todas las medidas en milímetros. Machos $\mathrm{N}=17$; $\operatorname{largo} \mathrm{X}=26.8(25.0-28.6)$; ancho $\mathrm{X}=3.28$ (3.0-3.5). Hembras $\mathrm{N}=12$; $\operatorname{largo} \mathrm{X}=30.2$ (27.333.5); ancho $\mathrm{X}=3.56(3.1-4.0)$.

Cabeza más ancha que el collum en hembras, de igual ancho que el collum en machos, de color castaño o castaño oscuro; genas densamente y cortamente pilosas. Cuerpo muy aplanado en ambos sexos; paraterguitos elevados. Collum castaño o castaño oscuro, con tres hileras transversales de macrosetas, la hilera anterior puede presentar de 6 a 9 macrosetas, la hilera media de 4 a 5 y la hilera posterior de 7 a 8 según el ejemplar; el collum también puede tener una o dos macrosetas a ambos lados del borde. Segmentos del cuerpo con prozonito y metaterguito castaño o castaño oscuro, paraterguitos castaño más claro. Segmentos del cuerpo también con tres hileras transversales de macrosetas, muy variables en cuanto al número de macrosetas por hilera; usualmente, la hilera anterior porta de 6 a 8 , la hilera media 4 a 6 y la hilera posterior 8 a 11; estas macrosetas nacen desde tubérculos prominentes o redondeados o directamente desde el dorso; los tubérculos dorsales están más o menos pronunciados hacia la región anterior del cuerpo. Segmentos no poríferos poseen adicionalmente 2 macrosetas en el margen lateral de cada paraterguito, naciendo desde diminutos tubérculos marginales; los segmentos poríferos sin macrosetas, pero con callo porífero prominente y alargado, el cual puede abarcar desde el diente del ángulo anterior del paraterguito hasta el ángulo posterior. Margen posterior de los metatergitos lisos, sin nódulos o procesos dentiformes. Ángulo anterior de los paraterguitos con diente bien marcado desde el segmento 2 al 8, diminuto desde el segmento 9 al 13 y ausente desde el segmento 14 hasta el final del cuerpo. Ángulo posterior de los paraterguitos de segmentos no poríferos muy agudo y pronunciado, proyectándose hacia atrás. Pleuritos y esternitos castaño claro, o pleuritos castaño oscuro y esternitos castaño claro. Antenas castaño claro u oscuro o algo anaranjadas. Patas castaño o algo amarillentas. Epiprocto castaño oscuro o con el ápice más claro; hypoprocto y valvas anales castaño oscuro. Machos con modificaciones sexuales secundarias que consisten en un prominente proceso cónico en las coxas del segundo par de patas y en procesos redondeados pilosos en las coxas del tercer par de patas.

Tipos. Holotipo macho 6669; paratipos machos 6589-6592, 6668, 6670-6680; machos juveniles 65936595, 6681-6684; hembras 6596-6603, 6685-6690; hembras juveniles 6604-6605, 6691; entre Sabana Real y Pirámide 204, a 4-5 km. de Sabana Real, al norte de Ángel Féliz y La Descubierta, Sierra de Neiba. República Dominicana; bajo piedras, hojarasca y troncos podridos; en bosque nublado; 5-XI-2006; cols. A. R. Pérez-Asso y H. Andújar. Todos los especímenes depositados en la colección ARPA (colección privada del autor).

Etimología. El epíteto específico alude a la localidad tipo (Sierra de Neiba).

Distribución geográfica. La especie se conoce solo de la localidad tipo (Fig.9).

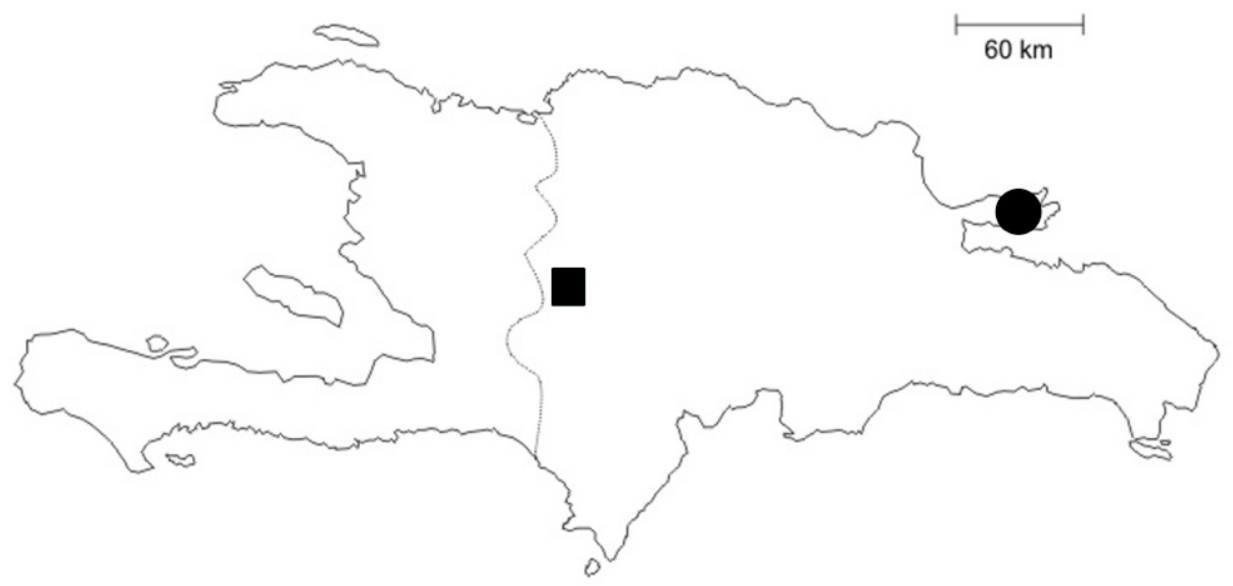


Nota. Como información adicional incluyo en este artículo la ilustración esquemática de la porción anterior del cuerpo de $P$. carinatus (Fig.2), así como la de los gonopodos en vista posterior (in situ) y en vista anterior (Figs.6-7).

\section{AGRADECIMIENTOS}

Mi gratitud a Yvonne Arias (Grupo Jaragua) por brindarnos las facilidades de hospedaje y transporte, así como por su colaboración en el estudio de la fauna de invertebrados de la República Dominicana. Mis agradecimientos a Celeste Mir y Carlos Suriel (Museo Nacional de Historia Natural, Santo Domingo) por brindarnos su apoyo y permitirnos el uso de las facilidades de dicha institución. A Héctor Andújar (Grupo Jaragua), quien colabora como chofer, guía y colector en las expediciones. Al personal de la Subsecretaría de Estado de Áreas Protegidas y Biodiversidad por facilitarnos los permisos de colecta en diversas regiones del país, en especial a Matilde Mota y Germán Dominici. Mi especial agradecimiento a Víctor González por el financiamiento de las expediciones y publicaciones y su interés en los estudios sobre la fauna de invertebrados de la República Dominicana.

\section{LITERATURA CITADA}

Loomis, H. F. 1941. Millipeds collected in Puerto Rico and the Dominican Republic by Dr. P. J. Darlington in 1938. Bull. Mus. Comp. Zool., 88 (2): 56-59, fig. 21.

Pérez-Asso, A. R. y D. E. Pérez-Gelabert. 2001. Checklist of the Millipeds (Diplopoda) of Hispaniola. Bol. S. E.A., 28: 75. 